\title{
Resuscitation Team Organization for Emergency Departments: A Concep- tual Review and Discussion
}

\author{
L.B. Mellick ${ }^{* 1}$ and B.D. Adams ${ }^{2}$ \\ ${ }^{I}$ Department of Emergency Medicine, Medical College of Georgia, Augusta, Georgia 30912, USA \\ ${ }^{2}$ Department of Clinical Investigation, William Beaumont Army Medical Center, 5005 North Piedras Street, El Paso, TX \\ 79920, USA
}

\begin{abstract}
In this article we discuss code or resuscitation team organization. The goals of this article are to define the questions surrounding code team organization and structure, discuss how organization can make a difference, review resuscitation systems and processes, and discuss aspects of team structure and performance. Issues of team performance include teamwork, leadership, communication and safety.
\end{abstract}

\section{INTRODUCTION}

\section{"And as it is Appointed unto Men Once to Die, but after} this the Judgment:" Heb 9:27 KJV

Whether the resuscitations occur in academic hospitals with level 1 trauma centers, rural hospitals, or long term nursing facilities, code team organization beyond a rudimentary level is often lacking. While some organizations have formalized their team structure, training schedules or team protocols are less common.

Increasingly, both the medical literature and resuscitation training materials emphasize code team organization [1-6]. Even though some health care providers remain skeptical and question the value of teamwork [3,7], the building evidence strongly supports code team organization as a worthwhile endeavor [8-14].

In the hospital setting resuscitative care is typically delivered by a variable number of individuals who almost always function within a team structure. The interventions of resuscitation are carried out by human agency and the quality of the resuscitation process is dependent on the agents delivering the care. Unfortunately, the agents delivering resuscitative care are vulnerable to error, fatigue, and distraction [15], and teamwork is a learned skill that does not occur naturally [16-20]. And, the quality and speed of care delivered during the resuscitation process can make a difference in patient outcome [12, 13, 21-25]. Examples include the decay of depth and rate of chest compressions over time that is reflected in patient morbidity and mortality [26-28], and the degradation in survival with delays in defibrillation of just seconds [22, 26-28].

This review necessarily focuses on resuscitation in the emergency department of the patient arriving via EMS. However, most aspects of team performance and communication described here are generalizable to both pre-hospital and to inpatient settings [7,29].

*Address correspondence to this author at the Department of Emergency Medicine, Medical College of Georgia, $112015^{\text {th }}$ Street, Augusta, Georgia 30912, USA; Tel: (706) 533-2931; Fax: (706) 364-2611;

E-mail: lmellick@mcg.edu

\section{MAKING A DIFFERENCE}

\section{Time and Outcomes}

In resuscitation literature the data is unequivocal: faster delivery of life saving interventions improves survival. A well organized EMS system saves lives [30-32]. We also know that the best survival rates occur in casinos and airline flights, not jogging paths or hospital lobbies [33-36]. Beneficial evidence of a timely team resuscitation in cardiac arrest as well as other critical conditions including trauma, stroke and sepsis is also strong [11, 13, 29, 37-40]. So the system response seems to matter more than the actual location [4144].

\section{Team Focus and Errors}

The systematic approach to a critically ill patient by a team competent group of nurses and physicians preserves a team's focus and prevents errors [45, 46]. Disagreements over procedures, confusion over team leadership, organizational chaos or demeaning comments represent poor team dynamics and can cause a team to lose focus. Team leaders often do not (or will not) follow basic protocols [47-49]. Medical errors tend to occur more frequently in stochastic environments or when team dynamics and communication are flawed [18, 50-53].

\section{Patient Safety}

The impact of code team organization on safety for both the patient and team members is an important consideration [27]. The research performed by the MedTeams project demonstrated improved patient safety in the emergency department setting [3]. Patient safety can be improved by specific organizational steps $[18,54-56]$. The growing trend of dedicated in-hospital cardiac arrest teams has demonstrated benefit, especially when systems are designed to alert teams to pre-arrest warning signs $[57,58]$.

\section{Stress Management}

A code potentially induces healthcare provider stress and may independently degrade performance [52, 59, 60]. Job satisfaction and longevity are directly linked to levels of 
work place stress [61-63]. With the code team, processes and procedures that are organized, disciplined and controlled are generally less stressful to the participants [60, 64-66]. The resuscitation team leader sets the tone for the team and prevents unnecessary stress levels [49].

\section{Family Presence}

In the context of increasing family presence, we have visitors and observers with highly emotional agendas and stakes. Despite depictions of chaotic resuscitations on television, the public's perceptions of cardiopulmonary resuscitations remain unrealistic $[67,68]$. Family members deserve to see a highly synchronized and disciplined pit crew at work $[69,70]$, and we should strive for resuscitations that function with no evidence of team dysfunction.

\section{RESUSCITATION PROCESSES}

Burkle and Rice first described the team process perspective [71]. The team perspective of a medical or trauma resuscitation involves at least seven phases. Each phase encompasses temporally important priorities for the resuscitation team.

\section{Anticipation Phase}

During this phase the data provided to the hospital by the paramedics is received and analyzed. Subsequently, the team is gathered, leadership is established, duties are delegated, the equipment is prepared and checked and the team members position themselves in readiness for the arrival of the critically ill patients.

\section{Entry Phase}

This involves the exchange of vital signs obtained by the paramedics just prior to their arrival. Additionally, there is the orderly transfer or exchange of the patient to the emergency department stretcher. The hospital resuscitation team members obtain baseline assessments of the patients A-BC's. The paramedics provide a concise history and new vital signs are obtained.

\section{Resuscitation Phase}

The team assesses the patients "A-B-Cs" (performs a primary survey) and carries out urgently indicated resuscitative interventions. Strong physician and nursing leadership are important during this phase. There should be one dominant voice and information is continuously provided to the team leadership. Vital signs are documented at least every five minutes and procedures and medication administration are accomplished. The secondary or anatomical survey is accomplished and when the patient's condition does not improve the physiologic survey is repeated. Effective communication between the team members and leadership is an important component of this phase.

\section{Maintenance Phase}

During this period of time the major assessment and resuscitative procedures have been accomplished. Continued stabilization of the patient is performed and intravenous lines and inserted catheters and tubes are stabilized. During this phase the team's "adrenaline rush" begins to subside as the most critical interventions have been accomplished. This is a vulnerable time period for the patient. A conscious effort to maintain the team's attention during this phase is an important responsibility of the team leadership.

\section{Family Notification Phase}

This is not a single point in time. In fact, the notification process continues throughout the resuscitation process. The resuscitation team designates at least one member to be a liaison with the family. Frequent, honest status reports and information updates are important to family members and should be carried out with sensitivity [72-75]. This is accomplished whether or not the family is physically present in the resuscitation room.

\section{Transfer Phase}

This phase does not necessarily occur at the end of a resuscitation. The potential for delays in necessary emergent care can occur if the transfer process is not managed efficiently. Remembering to arrange for transport late into resuscitation may not be efficient management of the patient's medical or surgical problem.

\section{Critique Phase}

Every resuscitation scenario is different and encounters unique problems. The patient care delivered and the team's interdependent performance should be critiqued as soon as possible after resuscitation. This allows maximal educational benefit from the process and prevents repeated inefficiencies with future patients. To varying degrees all resuscitation teams sustain emotional traumas following resuscitations and the process can be cumulative $[65,76,77]$. Debriefing or defusing processes can also occur during the critique phase that allows team members to begin to manage personal grief reactions. This process has been formalized as the critical incident stress debrief (CISD) borrowed from the disaster medicine experience [65, 76-78].

\section{JOB AIDS FOR CODE TEAMS}

Industries requiring high precision commonly utilize "job aids". Job aids can be checklists containing specific task elements for which accuracy and oversight prevention are mandatory. A classic example of a job aid is the preflight checklist used by airline pilots. The most common example of a job aid already used widely in resuscitation settings is the "ABC" (Airway-Breathing-Circulation) mnemonic for establishing patient resuscitation priorities. Other potential resuscitation job aids would be a room preparation checklist (See Fig. 1) or a wallboard (See Fig. 2) describing team member positions around the bed. Job aids may organize task priorities, standardize activities, prevent inadvertent oversights, allow faster and more thorough completion of tasks and reduce cognitive load [79-81]. They prevent leadership distractions or task drift, ensure a consistent structure to the resuscitation process and decrease team stress. Job aids transfer resuscitation processes from deliberate cognitive processes to automatic reflexes $[79,82,83]$.

There are other useful job aids besides checklists. Documents such as a contemporaneous trauma resuscitation record can increase the efficiency of documentation. Template order forms for critical diagnoses improve compliance with evidence based guidelines and patient outcomes [39, 84, 85]. Dry erase boards hung on the resuscitation room wall can assist with documentation of the prehospital history or 


\section{IDENTIFICATION OF TEAM MEMBER ROLES}

Documentation Nurse: Room preparation checklist, documentation, medication mixing, procedure trays, stays with patient.

Lead Nurse: Team preparation prior to arrival, delegates orders through RN from code team captain, traffic control, LEFT Nurse [Chest tube placement, peritoneal lavage, airway clearance], second to leave

- Circulating Nurse: RIGHT nurse [Intravenous line, draws blood, medication administration, NG, Foley placement, urine dip], first to leave

Technician: Places patient on monitors, performs manual BP, foley placement, EKG, Prep for procedures

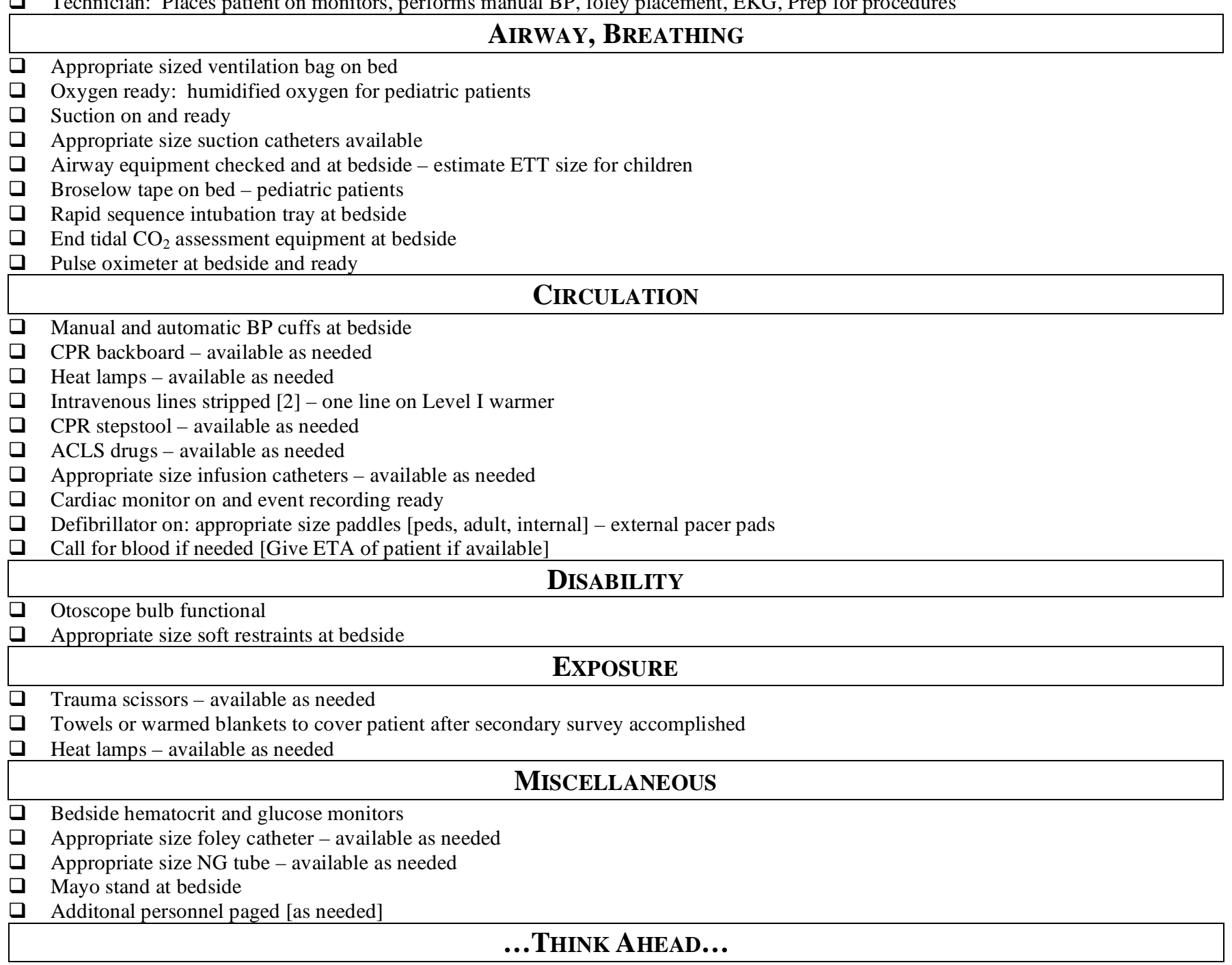

Are the appropriate invasive procedure supplies readily available??

Fig. (1). Room preparation checklist.

individuals responsible for team role assignments [81]. Posters containing reference information can be strategically placed on resuscitation room walls. Pediatric resuscitation drugs, the Glasgow Coma Scale, and ACLS algorithms are examples of information that are often posted. The roles, duties and locations of specific team members can also be displayed as a wall chart. Such a chart is especially useful in training center settings where team members regularly rotate.

Another important job aid is the Broselow ${ }^{\circledR} /$ Hinkle Pediatric Resuscitation System. The Broselow Tape is becoming a standard piece of equipment for most emergency departments. This tape allows the application of preset drug calculations and equipment sizes using an estimate of the child's weight based on the child's length. This system quickly and accurately estimates a child's weight, calculates drug doses and guides equipment size selection [79, 86].

Well thought out systems for equipment organization and display are also valuable to code team organization. The room location for equipment storage can affect resuscitation team efficiency. Minimize impediment of the floor space around the patient (especially near the head) by maximizing the use of overhead storage, x-ray, and outlets. Display airway equipment in a semi-open format on an airway wallboard placed at the head of the resuscitation bed. Many other equipment display options are available. Equipment cupboards with glass doors or open equipment carts allow easy visibility and access of supplies. Ergonomic design of resuscitation equipment from drug storage to defibrillator pads will definitely impact overall team efficiency [20, 87, 88]. 


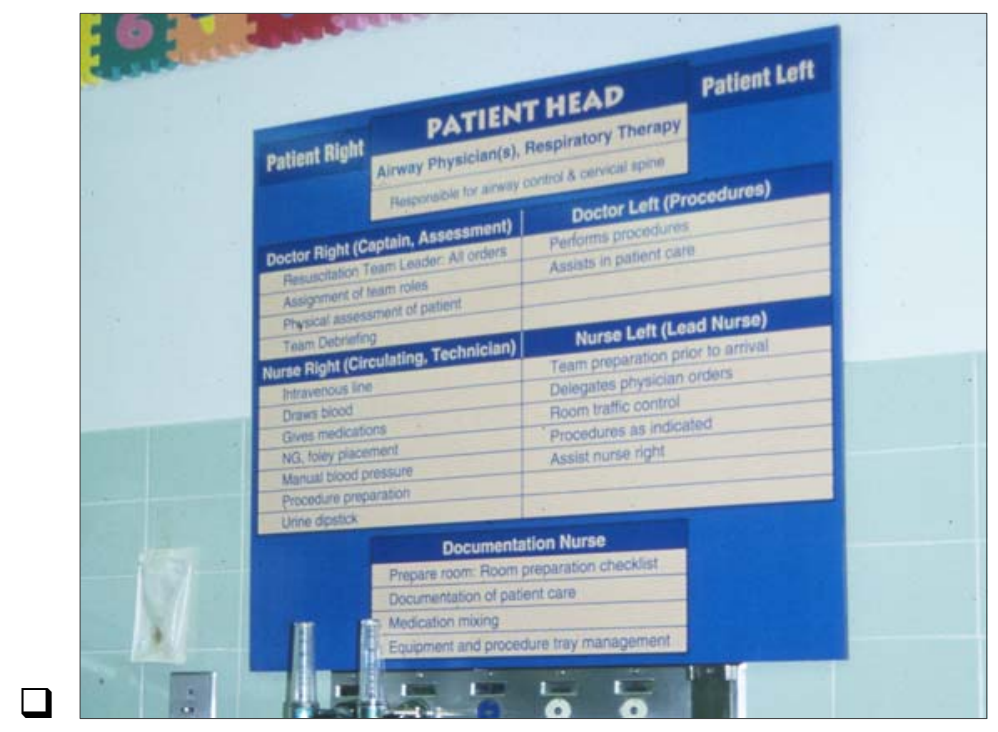

Fig. (2). Wallboard with resuscitation team room positions, roles and assigned activities.

While medical informatics show great promise in resuscitation team communications, a simple informative tracking board is valuable for managing multiple critically ill patients [81].

\section{TEAM DEVELOPMENT AND STRUCTURE}

In 1999, Risser introduced important team work concepts to the emergency department setting $[18,50,51,83,89]$. MedTeams is an adaptation and application of aviation flight crew resource management (CRM) to hospital emergency departments. The underlying premise for MedTeams was that most errors result from breakdowns in systems-level defenses and that a reduction in medical errors was possible through interdisciplinary teamwork. They identified five critical dimensions that were necessary for effective teamwork and identified specific, observable behaviors that were linked to these dimensions.

\section{Maintain Team Structure and Climate}

\section{Establish Leadership}

Clearly defined physician leadership is critical to the smooth performance of the code team [90]. There must be one and only one physician leader at any specific time who acts as the "sinus node" pacemaker for the resuscitation process? To prevent multi-focal and ineffective team leadership, no other "ectopic" leadership foci can be tolerated. The occurrence of a "discordant array of specialists each intent on an individual treatment plan" must be avoided [7, 91-94]. Well-defined but integrated nursing leadership is also critically important $[95,96]$.

Leadership is not always a natural process or event and requires certain finite actions to delineate the leadership role $[4,16,20,55,96,97]$. Steps or actions that assist in leadership declaration are listed below. Experienced leaders recognize the importance, the interdependence and the value of each team member. When the hierarchical structure becomes a barrier to communication, the benefit of leadership is diminished and patient safety is potentially compromised [98$102]$.

\section{Methods of Declaring Leadership \& Maintenance}

- Be present in the resuscitation room prior to the patient's arrival

- Assemble the team prior to the patient's arrival

- $\quad$ Lead the team in patient care planning prior to arrival of the patient

- Lead in the assignment and clarification of team member roles prior to the patient's arrival

- $\quad$ Position yourself in the room at a location typical for team leaders [at head or foot of bed]*

- Unitize your local system for team leader identification (colored gown, arm bands etc).

- $\quad$ Be the individual who directly receives the paramedic history

- $\quad$ Leadership admonition may be necessary and appropriate: "Take orders only from me."

- $\quad$ Act the part; decibels, rhythm, behavior and tone of voice should reinforce your role

- $\quad$ Establish identity of the team leader nurse and collaborate in resuscitation planning

- $\quad$ Enlist help of team leader nurse with team cooperation, crowd control and delegation of orders

- Make eye contact and address other team members by name

- Maintain ongoing communication that keeps the team continually informed 
*Business leaders have long known that room position signifies and even dictates leadership roles [103-107].

Establishing oneself as the leader is frequently an easier step than maintaining control of the resuscitation. Loss of control and confusion over team leadership can significantly impact on patient care delivery.

\section{Organize Team}

Team organization involves assigning roles, development of a treatment plan and preparing for the arrival of the patient. In this setting complexity can breed chaos. Therefore, the code team structure and organization should be natural, clinically relevant, easily reinforced and must augment rather than distract team member focus from the resuscitation.

Standardization of team member roles and room positions clarifies team member duties. (See Fig. 3) In reality, team member roles and room positions cannot be rigidly defined. Variations in room layout, location of monitors and equipment, number of personnel, available skill mix, patient size or age and the clinical condition being treated are factors that will influence roles and team member locations in the room [46]. Nevertheless, certain roles are assigned to specific room positions because of the activities performed or the location of major equipment [e.g. airway physician, cervical spine control, CPR administrator, defibrillator, cardiac monitors, and blood warmers]. Respiratory therapy, anesthesia or the emergency physician in charge of airway are positioned at the patient's head. Since physicians traditionally examine patients from the patient's right side, the team captain who often performs the physical assessment will usually occupy that location. During different phases of the resuscitation process and depending on the health condition being treated, the physician leadership location may transition.

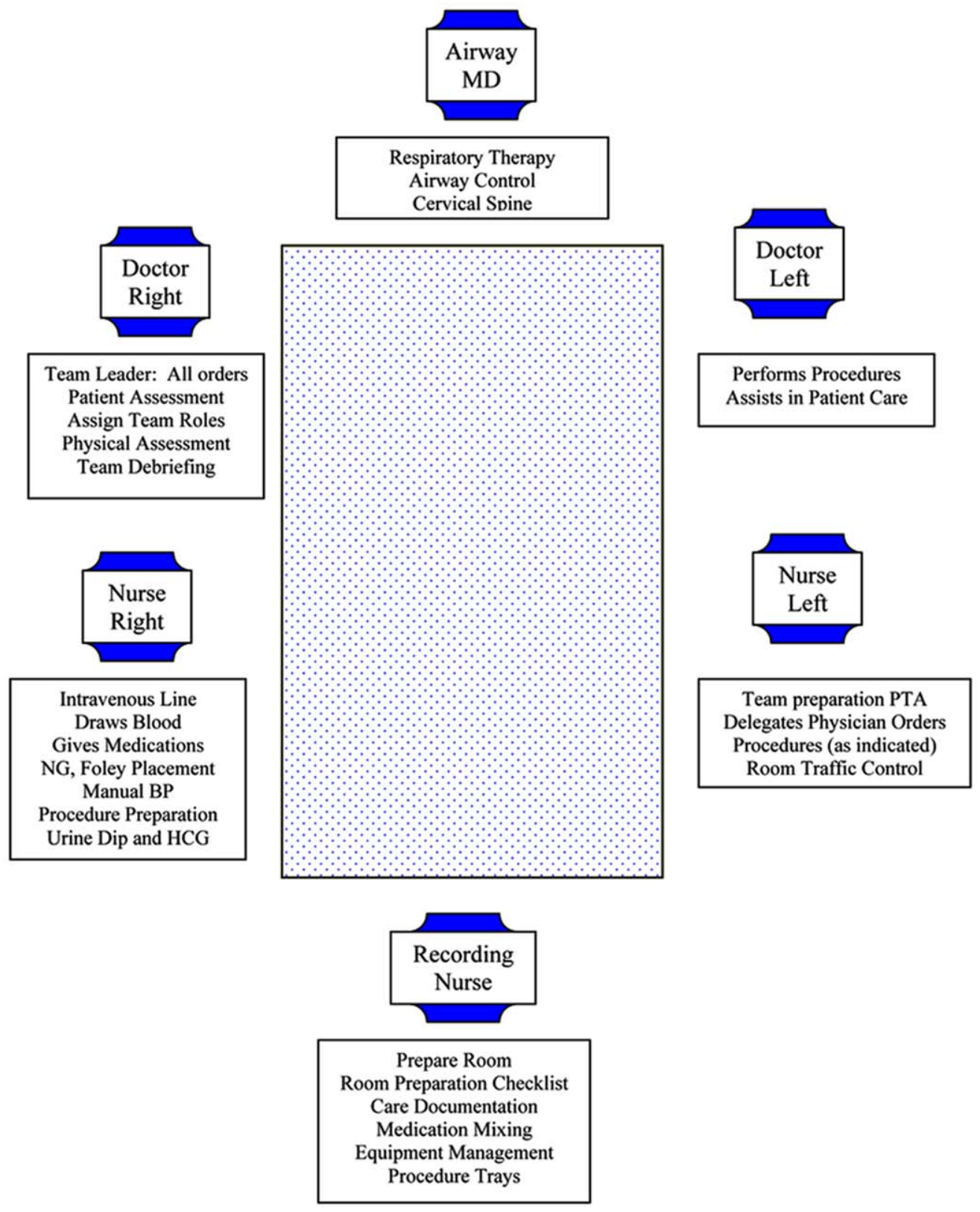

Fig. (3). Code team personnel positions and role descriptions. 
Concurrently, the lead nurse who under ideal circumstances is in continuous communication with the team captain should be located on the patient's left and across from the physician team leader. The location of other team members is dependent upon which procedures are being performed. The documentation nurse is typically stationed within eye contact and auditory proximity (which prevents having to request information) at a writing station or table. If a supply cart is located immediately behind and a drug cart nearby, the documentation nurse can simultaneously assist with equipment and medication distribution. A wallboard can effectively direct the team members to their room locations as well as prompt concerning the duties of their assigned role (See Fig. 2).

Compared to the academic medical center the rural emergency department will have different resources [48, 98, 99]. In smaller emergency departments the team captain is often responsible for the airway and the performance of other time and attention consuming procedures. In this setting the need for preplanning and team organization is even greater and it in no way diminishes the need for code team organization. Smaller hospitals can create emergency department response teams to augment personnel resources and allow team management of critically ill patients [12, 23, 90].

Increasingly, modern hospitals pre-designate specific wards or teams to help perform specific therapeutic and sometimes resuscitation functions. Examples include CPR team, Trauma Team, Chest pain units [CPU], cardiac care units (CCU) and more recently Rapid Response Teams [10, 37, 108]. Because many resuscitations occur after admission to the hospital, the team roles of CPU, CCU and RRT are essential to satisfactory patient outcomes. The general principles of emergency department teamwork and team organization should be applicable to these specific units as well.

\section{Cultivate Team Climate and Resolve Conflicts Construc- tively}

Effective resuscitation team performance can be visualized as an auto racing pit-crew. The effective resuscitation team shares with the auto racing pit-crew the characteristics of well defined leadership, clearly identified responsibilities, specific role delineations, an emphasis on efficiency and speed, expert accomplishment of individual roles, team performance and dynamics, adequate training and preparation, well delineated priorities and an appropriate number of team members. Team dynamics of a pit-crew also include performance as a unified, concerted and harmonious effort that is carefully choreographed for flawless timing.

A team's mission should not be distracted by internal competition and team energies should be directed to the common external goal. Unfortunately, the resuscitation setting is often the place where different specialty cultures interface. Anesthesiologists, surgeons and emergency physicians participating in the care of the same patient can experience barriers to team unity due to specialty rivalry, sense of entitlement, or differing style of communication [52, 89].

Resuscitations are potentially emotionally and intellectually demanding settings. Because of these performance demands, mistakes in team ambiance sometimes occur. Angry tantrums do little to improve patient care or increase team efficiency and effectiveness. The team leader is primarily responsible for establishing a healthy team ambiance. On occasion, it is appropriate for a team leader to dismiss an inappropriate team member from the resuscitation suite.

\section{Apply Problem Solving Strategies}

\section{Conduct Situational Planning}

The team leader should involve the team in the planning process prior to the patient's arrival. A brief review of the expected resuscitation procedures, protocols and required resources should be accomplished. A room preparation checklist may also be used to insure that standard preparation steps have been accomplished (See Fig. 1).

\section{Apply Decision Making Methods}

Team members must insure that the team leader has all pertinent clinical information. At the same time the physician leader of the resuscitation must not hesitate to engage team members in decision making; asking for direction or suggestions when necessary.

\section{Engage in Error Avoidance Actions}

Communication techniques that promote error avoidance such as the "check back", the "call out", the "hand-off" and the "two challenge rule" are used. With the check-back system the receiver of a verbal order is required to repeat that order and receive verification before a drug is administered. The "call out" is a request for information input by the team leader. The "hand off" rule is turning over leadership responsibility whenever indicated such as when a procedure is performed by the team leader. The "Two Challenge Rule", a technique borrowed from aviation, is also used by the team when necessary. With the "Two Challenge Rule" team members are responsible for questioning all actions that might place the patient at risk. A challenge is voiced at least twice to assure it has been heard. The receiver must acknowledge the challenge and offer an explanation for the action in question [5].

Monitoring the teamwork process also protects against errors. Cross monitoring of team member actions should be ongoing and observed deviations from the established protocols are challenged. The process of cross monitoring allows a common situational awareness to be developed [5, 81]. This allows recognition of impending errors as well as better-coordinated activities. The team should be immediately alerted to errors and corrective actions must be advocated for and rapidly taken.

\section{Communicate with the Team}

Ongoing and effective communication by the team allows its members to establish a common reference and understanding of patient and operational issues [89]. Team members continually feed information to the team leader, who analyzes, makes decisions and responds back with further direction. When giving directions or orders, the team leader assigns responsibility as well. Calling orders out without assigning responsibility produces one of two nonproductive responses; too many team members respond or no one responds. An effective resuscitation team also communicates through non-verbal as well as verbal methods $[61,78$, 109]. Characteristics of good team communication techniques are listed below. 


\section{Characteristics of Good Team Communication}

- The team has a clear chain of command and communication focuses on the team leader

- $\quad$ The leader effectively applies the team resources and gives consistent guidance to the team

- $\quad$ Team leader nurse receives orders from team captain and delegates them to team members

- $\quad$ No communication barriers caused by the team's hierarchy or chain of command

- $\quad$ Team leader recognizes his/her interdependent relationships with other team members

- Orders given directly to individuals, addressed by name along with eye contact

- $\quad$ Speed of communication is important to code team control

- $\quad$ The team leader is the team pacemaker

- Clarity and efficiency of communication

- Appropriate loudness or decibels of speech

- $\quad$ Effective non-verbal communication occurs because individuals follow sequential protocol, anticipate and monitor the team leader and other team members

- $\quad$ Team leaders encourage questions and suggestions

- Team leader has continuous conversation with the team providing current information

- Team has common operational framework and vocabulary

\section{Execute Plans, Cross Monitor and Manage Workloads}

Avoid circumstances that increase the risk of error or decay in team performance. Team members are responsible for requesting assistance when task overload exists or offering teammates assistance when they become aware of overload situations. Physician and nurse leadership are responsible for insuring that the workload within the team is balanced [81]. If team members assist team members, clinical errors caused by individual overload, stress or distractions will be reduced.

\section{Primary and Secondary Triage}

The team leader establishes, prioritizes and directs the therapeutic plan for the patient. The team leader solicits and considers individual team member observations and assessments. With this information the team captain initiates adjustments in patient care in a real-time fashion. Additional resources are obtained as needed. When the patient's condition deteriorates, the team returns to the "ABCs".

\section{Maintain Situational Awareness}

Situational awareness is a responsibility of the entire team, and all team members strive to maintain an overall awareness of the resuscitation process. As part of that process the team leader should provide an ongoing and clearly stated report of the process so that the team is kept abreast of each stage of the resuscitation. For example, "We are now starting the secondary survey and the C-spine films are back and are negative." All team members monitor the actions of others on the team for compliance with protocol, equitable workload distribution and errors of omission or commission.

\section{Improve Team Skill}

\section{Engage in Informal Team Improvement Strategies}

Even in a busy emergency department setting the critique phase of the resuscitation process can be accomplished and can prove to be a valuable training experience [71]. Debriefing or personal defusing, especially utilizing the critical incident debriefing system can also occur during the critique phase allowing team members to manage personal grief reactions $[61,65]$.

\section{Engage in Formal Team Improvement Strategies}

Advanced life support training using mock codes can improve team performance and ultimately even survival outcomes $[91,110,111]$. Actual training utilizing realistic code team organization is an important component of any ALS program [112]. A code organization committees or similar entity can be another important improvement strategy.

\section{Training and Mock Codes}

Mock codes are an ideal tool for resuscitation team training. There should be two areas of focus for mock code training. The first is the standard "megacode" in which ATLS and ACLS clinical scenarios are practiced and reviewed $[113,114]$. A broad spectrum of resuscitation situations involving simulated patients of varying ages and conditions should be incorporated [113]. Alternatives to intravenous access and difficult airway management are included in this training. The second area of training emphasis is code team organization. While formal systems for teaching the clinical aspects of resuscitation are common, training in code team organization tends to occur much less frequently. Attention to both aspects of training is important.

\section{Code Organization Committee}

There are several formats that allow effective administration of code team organization. Administrative management of code organization can be accomplished in subcommittees of hospital trauma, CPR, morbidity and mortality committees, or emergency department QA committee meetings $[112,115]$. While there are many universal issues, resuscitation team planning should be accomplished in the context of each individual hospital following the Utstein guidelines $[113,114,116]$.

\section{Code Committee Activities}

- $\quad$ Organize the ED resuscitation team process

- Define team membership or composition

- Establish team member credentialing and training criteria (ACLS, ATLS, PALS, etc).

- $\quad$ Plan specific team roles and assignments

- Review any team performance problems (Not QA)

- Interface with team members who fail to understand team dynamics

- Identify quality assurance issues and refer to appropriate QA committee 
- $\quad$ Plan and orchestrate training activities

- $\quad$ Supervise training

- Establish documentation standards according to the Utstein template

- $\quad$ Supervise new equipment procurement and training

- $\quad$ Oversee equipment stocking and display

- Identify and correct logistical and supply problems

- Interface with other services or departments when necessary

- Identify areas for resuscitation research

- Institute training in notification of next of kin

- Create code organization benchmarks

- $\quad$ Establish benchmarks for timeliness of admission or transfer

- Seamless movement of patient within the institution

- $\quad$ Prehospital educational role

\section{CONCLUSION}

In this article the case for a greater focus on code team organization has been presented. Lack of organization may result in unwanted consequences for others. While it may be "appointed unto men once to die" it is possible that a second opportunity may be afforded to more patients if their resuscitation teams arrive sooner, are better organized and are more efficient in their delivery of life saving interventions.

\section{REFERENCES}

[1] Anonymous. AARC (American Association for Respiratory Care) clinical practice guideline. Defibrillation during resuscitation. Respir Care 1995; 40(7): 744-8.

[2] Cummins RO, Sanders A, Mancini E, Hazinski MF. In-hospital resuscitation: executive summary. Ann Emerg Med 1997; 29(5): 647-9.

[3] Morey JC, Simon R, Jay GD, et al. Error reduction and performance improvement in the emergency department through formal teamwork training: evaluation results of the MedTeams project. Health Serv Res 2002; 37(6): 1553-81.

[4] Ostergaard HT, Ostergaard D, Lippert A. Implementation of team training in medical education in Denmark. Qual Saf Health Care 2004; 13(Suppl 1): i91-5.

[5] Small SD, Wuerz RC, Simon R, Shapiro N, Conn A, Setnik G. Demonstration of high-fidelity simulation team training for emergency medicine. Acad Emerg Med 1999; 6(4): 312-23.

[6] Anonymous. ACLS Provider Manual Dallas: American Heart Association 2001.

[7] Soar J, McKay U. A revised role for the hospital cardiac arrest team? Resuscitation 1998; 38(3): 145-9.

[8] Becker LB, Pepe PE. Ensuring the effectiveness of communitywide emergency cardiac care. Ann Emerg Med 1993; 22(2 Pt 2): 354-65.

[9] Sandroni C, Cavallaro F, Ferro G, et al. A survey of the in-hospital response to cardiac arrest on general wards in the hospitals of Rome. Resuscitation 2003; 56(1): 41-7.

[10] Adams BD, Zeiler K, Jackson WO, Hughes B. Emergency medicine residents effectively direct inhospital cardiac arrest teams. Am J Emerg Med 2005; 23(3): 304-10.

[11] DeVita MA, Braithwaite RS, Mahidhara R, et al. Use of medical emergency team responses to reduce hospital cardiopulmonary arrests. Qual Saf Health Care 2004; 13(4): 251-4.

[12] Taylor SF, Gerhardt RT, Simpson MP. An association between emergency medicine residencies and improved trauma patient outcome. J Emerg Med 2005; 29(2): 123-7.
[13] Henderson SO, Ballesteros D. Evaluation of a hospital-wide resuscitation team: does it increase survival for in-hospital cardiopulmonary arrest? Resuscitation 2001; 48(2): 111-6.

[14] Peberdy MA, Kaye W, Ornato JP, et al. Cardiopulmonary resuscitation of adults in the hospital: a report of 14720 cardiac arrests from the national registry of cardiopulmonary resuscitation. Resuscitation 2003; 58(3): 297-308.

[15] Reason J. Human error: models and management. BMJ 2000; 320(7237): 768-70.

[16] Leape LL. A systems analysis approach to medical error. J Eval Clin Pract 1997; 3(3): 213-22.

[17] Thomas EJ, Sexton JB, Helmreich RL. Translating teamwork behaviours from aviation to healthcare: development of behavioural markers for neonatal resuscitation. Qual Saf Health Care 2004; 13(Suppl 1): i57-64.

[18] Risser D, Simon R, Rice M, Salisbury M. A structured teamwork system to reduce clinical errors. In: Spath P, Ed. error reduction in health care: a systems approach to improving patient safety. San Francisco: Jossey-Bass 2000.

[19] Jevon P. Resuscitation in hospital: resuscitation council (UK) recommendations. Nurs Stand 2002; 16(33): 41-4.

[20] Risser DT, Rice MM, Salisbury ML, Simon R, Jay GD, Berns SD. The potential for improved teamwork to reduce medical errors in the emergency department. The MedTeams Research Consortium. Ann Emerg Med 1999; 34(3): 373-83.

[21] Jacobs I, Callanan V, Nichol G, et al. The chain of survival. Ann Emerg Med 2001; 37(Suppl 4): S5-16.

[22] Spearpoint KG, McLean CP, Zideman DA. Early defibrillation and the chain of survival in 'in-hospital' adult cardiac arrest; minutes count. Resuscitation 2000; 44(3): 165-9.

[23] Porter JM, Ursic C. Trauma attending in the resuscitation room: does it affect outcome? Am Surg 2001; 67(7): 611-4.

[24] Sandroni C, Ferro G, Santangelo S, et al. In-hospital cardiac arrest: survival depends mainly on the effectiveness of the emergency response. Resuscitation 2004; 62(3): 291-7.

[25] Zafari AM, Zarter SK, Heggen V, et al. A program encouraging early defibrillation results in improved in-hospital resuscitation efficacy. J Am Coll Cardiol 2004; 44(4): 846-52.

[26] Chan PS, Krumholz HM, Nichol G, et al. Delayed time to defibrillation after in-hospital cardiac arrest. N Engl J Med 2008; 358(1): 9-17.

[27] Adams BD. Code Blue-Where To? Rockville,: Agency for Healthcare Research and Quality; 2007 (updated 2007 15 Jan 2008; cited); Available from: http://www.webmm.ahrq.gov/case.aspx? caseID $=162$

[28] Anonymous. Guidelines 2000 for cardiopulmonary resuscitation and emergency cardiovascular care. Part 4: the automated external defibrillator: key link in the chain of survival. The American heart association in collaboration with the international liaison committee on resuscitation. Circulation 2000; 102(Suppl 8): 160-76.

[29] Buist MD, Moore GE, Bernard SA, Waxman BP, Anderson JN, Nguyen TV. Effects of a medical emergency team on reduction of incidence of and mortality from unexpected cardiac arrests in hospital: preliminary study. BMJ 2002; 324(7334): 387-90.

[30] Sanders AB, Ewy GA. Cardiopulmonary resuscitation in the real world: when will the guidelines get the message? JAMA 2005; 293(3): 363-5.

[31] Rea TD, Eisenberg MS, Becker LJ, et al. Emergency medical services and mortality from heart disease: a community study. Ann Emerg Med 2003; 41(4): 494-9.

[32] Stiell IG, Wells GA, DeMaio VJ, et al. Modifiable factors associated with improved cardiac arrest survival in a multicenter basic life support/defibrillation system: OPALS study phase I results. Ontario Prehospital Advanced Life Support. Ann Emerg Med 1999; 33(1): 44-50.

[33] Gratton M, Lindholm DJ, Campbell JP. Public-access defibrillation: where do we place the AEDs? Prehosp Emerg Care 1999; 3(4): 303-5.

[34] Valenzuela TD, Roe DJ, Nichol G, Clark LL, Spaite DW, Hardman RG. Outcomes of rapid defibrillation by security officers after cardiac arrest in casinos. N Engl J Med 2000; 343(17): 1206-9.

[35] Page RL, Joglar JA, Kowal RC, et al. Use of automated external defibrillators by a U.S. airline. N Engl J Med 2000; 343(17): 12106.

[36] Zipes DP. Saving time saves lives. Circulation 2001; 104(21): 2506-8. 
[37] Halvorsen L, Garolis S, Wallace-Scroggs A, et al. Building a rapid response team. AACN Adv Crit Care 2007; 18(2): 129-40.

[38] Blow O, Magliore L, Claridge JA, Butler K, Young JS. The golden hour and the silver day: detection and correction of occult hypoperfusion within 24 hours improves outcome from major trauma. J Trauma 1999; 47(5): 964-9.

[39] Rivers E, Nguyen B, Havstad S, et al. Early goal-directed therapy in the treatment of severe sepsis and septic shock. N Engl J Med 2001; 345(19): 1368-77.

[40] Stroke Unit Trialists Collaboration. Organised inpatient (stroke unit) care for stroke. (update of Cochrane Database Syst Rev 2000; (2): CD000197). Cochrane Database Syst Rev 2002; (1): CD000197.

[41] Skrifvars MB, Saarinen K, Ikola K, Kuisma M. Improved survival after in-hospital cardiac arrest outside critical care areas. Acta Anaesthesiol Scand 2005; 49(10): 1534-9.

[42] Myerburg RJ, Velez M, Rosenberg DG, Fenster J, Castellanos A. Automatic external defibrillators for prevention of out-of-hospital sudden death: effectiveness of the automatic external defibrillator. $\mathbf{J}$ Cardiovasc Electrophysiol 2003; 14(Suppl 9): S108-16.

[43] Adams BD. Cardiac arrest of nonpatients within hospital public areas. Am J Cardiol 2005; 95(11): 1370-1.

[44] Cummins RO, Chamberlain DA, Abramson NS, et al. Recommended guidelines for uniform reporting of data from out-ofhospital cardiac arrest: the Utstein Style. A statement for health professionals from a task force of the American heart association, the European resuscitation council, the heart and stroke foundation of Canada, and the Australian resuscitation council. Circulation 1991; 84(2): 960-75.

[45] Xiao Y, Mackenzie CF, Eds. Collaboration in complex medical systems. North Atlantic Treaty Organization Human Factors Meeting Symposium 1998.

[46] Xiao Y, Seagull FJ, Mackenzie CF, Klein KJ, Ziegert JC. Adaptation of team structure of trauma resuscitation teams. Proc Human Factors Ergon Soc 46th Ann Meeting 2002; pp. 569-73.

[47] DeAnda A, Gaba DM. Role of experience in the response to simulated critical incidents. Anesth Analg 1991; 72(3): 308-15.

[48] Cline DM, Welch KJ, Cline LS, Brown CK. Physician compliance with advanced cardiac life support guidelines. Ann Emerg Med 1995; 25(1): 52-7.

[49] Hoff WS, Reilly PM, Rotondo MF, DiGiacomo JC, Schwab CW. The importance of the command-physician in trauma resuscitation. J Trauma 1997; 43(5): 772-7.

[50] Kohn L, Corrigan J, Donaldson M. Why do errors happen. to err is human: building a safer health system, Washington, DC: Institute of Medicine 2000

[51] Spencer FC. Human error in hospitals and industrial accidents: current concepts. J Am Coll Surg 2000; 191(4): 410-8.

[52] LaFasto FMJ, Larson CE. When teams work best. Thousand Oaks: Sage 2001.

[53] Clarke JR, Spejewski B, Gertner AS, et al. An objective analysis of process errors in trauma resuscitations. Acad Emerg Med 2000; 7(11): 1303-10.

[54] White AA, Wright SW, Blanco R, et al. Cause-and-effect analysis of risk management files to assess patient care in the emergency department. Acad Emerg Med 2004; 11(10): 1035-41.

[55] Amalberti R, Auroy Y, Berwick D, Barach P. Five system barriers to achieving ultrasafe health care. Ann Intern Med 2005; 142(9): 756-64.

[56] Schenkel S. Promoting patient safety and preventing medical error in emergency departments. Acad Emerg Med 2000; 7(11): 120422.

[57] Buist M, Harrison J, Abaloz E, et al. Six year audit of cardiac arrests and medical emergency team calls in an Australian outer metropolitan teaching hospital. BMJ 2007; 335(7631): 1210-2.

[58] Kenward G, Robinson A, Bradburn S, et al. False cardiac arrests: the right time to turn away? Postgrad Med J 2007; 83(979): 344-7.

[59] Morgan R, Westmoreland C. Survey of junior hospital doctors' attitudes to cardiopulmonary resuscitation. Postgrad Med J 2002; 78(921): 413-5.

[60] Schull MJ, Ferris LE, Tu JV, Hux JE, Redelmeier DA. Problems for clinical judgement: 3 . Thinking clearly in an emergency. CMAJ 2001; 164(8): 1170-5.

[61] Laposa JM, Alden LE, Fullerton LM. Work stress and posttraumatic stress disorder in ED nurses/personnel. J Emerg Nurs 2003; 29(1): 23-8.
[62] Knazik SR, Gausche-Hill M, Dietrich AM, et al. The death of a child in the emergency department. Ann Emerg Med 2003; 42(4): 519-29.

[63] Whitley TW, Gallery ME, Allison EJ, Jr, Revicki DA. Factors associated with stress among emergency medicine residents. Ann Emerg Med 1989; 18(11): 1157-61.

[64] Schaefer HG, Helmreich RL, Scheidegger D. Human factors and safety in emergency medicine. Resuscitation 1994; 28(3): 221-5.

[65] Laws T. Examining critical care nurses' critical incident stress after in hospital cardiopulmonary resuscitation (CPR). Aust Crit Care 2001; 14(2): 76-81.

[66] Featherstone P, Smith GB, Linnell M, Easton S, Osgood VM. Impact of a one-day inter-professional course (ALERT) on attitudes and confidence in managing critically ill adult patients. Resuscitation 2005; 65(3): 329-36.

[67] Diem SJ, Lantos JD, Tulsky JA. Cardiopulmonary resuscitation on television. Miracles and misinformation. N Engl J Med 1996; 334(24): 1578-82.

[68] Jones GK, Brewer KL, Garrison HG. Public expectations of survival following cardiopulmonary resuscitation. Acad Emerg Med 2000; 7(1): 48-53.

[69] Schmidt TA, Norton RL, Tolle SW. Sudden death in the ED: educating residents to compassionately inform families. J Emerg Med 1992; 10(5): 643-7.

[70] Boyd R. Witnessed resuscitation by relatives. Resuscitation 2000; 43(3): 171-6.

[71] Burkle FM, Rice MM. Code organization. Am J Emerg Med 1987; 5(3): 235-9.

[72] Critchell CD, Marik PE, Critchell CD, Marik PE. Should family members be present during cardiopulmonary resuscitation? A review of the literature. Am J Hosp Palliat Care 2007; 24(4): 311-7.

[73] Kuzin JK, Yborra JG, Taylor MD, et al. Family-member presence during interventions in the intensive care unit: perceptions of pediatric cardiac intensive care providers. Pediatrics 2007; 120(4): e895-901.

[74] Foresman-Capuzzi J, Foresman-Capuzzi J. Grief telling: death of a child in the emergency department. J Emerg Nurs 2007; 33(5): 505-8.

[75] Fulbrook P, Latour J, Albarran J, et al. The presence of family members during cardiopulmonary resuscitation: European federation of critical care nursing associations, European society of paediatric and neonatal intensive care and European society of cardiology council on cardiovascular nursing and allied professions joint position statement. Nurs Crit Care 2007; 12(5): 250-2.

[76] Jiggetts SM, Hall DP, Jr. Helping the helper: $528^{\text {th }}$ combat stress center in Somalia. Mil Med 1995; 160(6): 275-7.

[77] Linton JC, Kommor MJ, Webb CH. Helping the helpers: the development of a critical incident stress management team through university/community cooperation. Ann Emerg Med 1993; 22(4): 6638.

[78] Smith A, Roberts K. Interventions for post-traumatic stress disorder and psychological distress in emergency ambulance personnel: a review of the literature. Emerg Med J 2003; 20(1): 75-8.

[79] Luten R, Wears RL, Broselow J, Croskerry P, Joseph MM, Frush $\mathrm{K}$. Managing the unique size-related issues of pediatric resuscitation: reducing cognitive load with resuscitation aids. Acad Emerg Med 2002; 9(8): 840-7.

[80] Ward P, Johnson LA, Mulligan NW, Ward MC, Jones DL. Improving cardiopulmonary resuscitation skills retention: effect of two checklists designed to prompt correct performance. Resuscitation 1997; 34(3): 221-5.

[81] Xiao Y. Artifacts and collaborative work in healthcare: methodological, theoretical, and technological implications of the tangible. $\mathrm{J}$ Biomed Inform 2005; 38(1): 26-33.

[82] Croskerry P. The importance of cognitive errors in diagnosis and strategies to minimize them. Acad Med 2003; 78(8): 775-80.

[83] Nolan TW. System changes to improve patient safety. BMJ 2000; 320(7237): 771-3.

[84] Eagle KA, Montoye CK, Riba AL, et al. Guideline-based standardized care is associated with substantially lower mortality in medicare patients with acute myocardial infarction: the American college of cardiology's guidelines applied in practice (GAP) projects in michigan. J Am Coll Cardiol 2005; 46(7): 1242-8.

[85] Sinuff T, Cook DJ, Randall J, Allen CJ. Evaluation of a practice guideline for noninvasive positive-pressure ventilation for acute respiratory failure. Chest 2003; 123(6): 2062-73. 
[86] Shah AN, Frush K, Luo X, Wears RL. Effect of an intervention standardization system on pediatric dosing and equipment size determination: a crossover trial involving simulated resuscitation events. Arch Pediatr Adolesc Med 2003; 157(3): 229-36.

[87] Telesca K. A simplistic approach to restocking crash carts. Hosp Pharm 1992; 27(12): 1068-70.

[88] Adams BD, Easty DM, Stuffel E, Hartman I. Decreasing the time to defibrillation: a comparative study of defibrillator electrode designs. Resuscitation 2005; 66(2): 171-4.

[89] Spath P. Reducing errors through work system improvements. In: Spath P, Ed. Error reduction in health care. San Francisco: JosseyBass 2000; pp. 199-234.

[90] Ummenhofer W, Amsler F, Sutter PM, Martina B, Martin J, Scheidegger D. Team performance in the emergency room: assessment of inter-disciplinary attitudes. Resuscitation 2001; 49(1): 39-46.

[91] Brown J, Latimer-Heeter M, Marinelli A, Rex E, Reynolds L. The first 3 minutes: code preparation for the staff nurse. Orthop Nurs 1995; 14(3): 35-40.

[92] Fields ML. The C.P.R. team in a medium-sized hospital. Am J Nurs 1966; 66(1): 87-90.

[93] Dane FC, Russell-Lindgren KS, Parish DC, Durham MD, Brown TD. In-hospital resuscitation: association between ACLS training and survival to discharge. Resuscitation 2000; 47(1): 83-7.

[94] Mohr JJ, Batalden PB. Improving safety on the front lines: the role of clinical microsystems. Qual Saf Health Care 2002; 11(1): 45-50.

[95] Gilligan P, Bhatarcharjee C, Knight G, et al. To lead or not to lead? Prospective controlled study of emergency nurses' provision of advanced life support team leadership. Emerg Med J 2005; 22(9): 628-32.

[96] Cooper S, Wakelam A. Leadership of resuscitation teams: lighthouse leadership. Resuscitation 1999; 42(1): 27-45.

[97] Hayes CW, Rhee A, Detsky ME, et al. Residents feel unprepared and unsupervised as leaders of cardiac arrest teams in teaching hospitals: a survey of internal medicine residents. Crit Care Med 2007; 35(7): 1668-72.

[98] Sanders AB, Berg RA, Burress M, Genova RT, Kern KB, Ewy GA. The efficacy of an ACLS training program for resuscitation from cardiac arrest in a rural community. Ann Emerg Med 1994; 23(1): 56-9.

[99] Camp BN, Parish DC, Andrews RH. Effect of advanced cardiac life support training on resuscitation efforts and survival in a rural hospital. Ann Emerg Med 1997; 29(4): 529-33.

[100] Malay ME, Moore JF. Rural-urban partnering in continuing education. J Contin Educ Nurs 2002; 33(2): 60-2.

[101] Cosby KS, Croskerry P. Profiles in patient safety: authority gradients in medical error. Acad Emerg Med 2004; 11(12): 1341-5.

[102] Croskerry P. The feedback sanction. Acad Emerg Med 2000; 7(11): 1232-8.
[103] Giesen M, McClaren HA. Discussion, distance and sex: changes in impressions and attraction during small group interaction. Sociometry 1976; 39(1): 60-70.

[104] Nova N. A review of how space affords socio-cognitive processes during collaboration. Psychnol J 2005; 3(2): 118-48.

[105] Sommer R. Further studies of small group ecology. Sociometry 1965; 28(4): 337-48.

[106] O'Hagan Lobb M. Seating arrangement as a predictor of small group interaction. J Adv Nurs 1982; 7(2): 163-6.

[107] Mitchell JT. Development and functions of a critical incident stress debriefing team. J Emerg Med Serv 1988; 12: 42-6.

[108] Cassin M, Macor F, Cappelletti P, et al. Management of patients with low-risk chest pain at the time of admission: a prospective study on a non-selected population from the Emergency Department Italian Heart Journal. Official J Ital Fed Cardiol 2002; 3(7): 399-405.

[109] Mitchell JT. Stress development and functions of a critical incident stress debriefing team. J Emerg Med Serv JEMS 1988; 13(12): 426.

[110] Whitcomb J, Brierton D, Kuhagen K, et al. Code teams and the review of cardiac arrests. Qual Rev Bull 1990; 16(4): 156-62.

[111] Kaye W, Mancini ME. Use of the Mega Code to evaluate team leader performance during advanced cardiac life support. Crit Care Med 1986; 14(2): 99-104.

[112] Whitcomb J, Brierton D, Kuhagen K, et al. Code teams and the review of cardiac arrests. Qual Rev Bull 1990; 16(4): 156-62.

[113] Granneman S, Conn VS. An evaluation of the effectiveness of competency-based code blue education. J Nurs Staff Dev 1996; 12(6): 283-8.

[114] Cappelle C, Paul RI. Educating residents: the effects of a mock code program. Resuscitation 1996; 31(2): 107-11.

[115] Anonymous. Management of Information. Hospital accreditation standards accreditation policies standards intent statements. Oakbrook Terrace, IL: The Joint Commission on Accreditation of Healthcare Organizations 2002; pp. 254-5.

[116] Jacobs I, Nadkarni V, Bahr J, et al. Cardiac arrest and cardiopulmonary resuscitation outcome reports: update and simplification of the Utstein templates for resuscitation registries: a statement for healthcare professionals from a task force of the International liaison committee on resuscitation (American heart association, European resuscitation council, Australian resuscitation council, New Zealand resuscitation council, heart and stroke foundation of Canada, InterAmerican heart foundation, resuscitation councils of Southern Africa). Circulation 2004; 110(21): 3385-97.

(C) Mellick and Adams; Licensee Bentham Open.

This is an open access article licensed under the terms of the Creative Commons Attribution Non-Commercial License (http://creativecommons.org/licenses/by-nc/3.0/) which permits unrestricted, non-commercial use, distribution and reproduction in any medium, provided the work is properly cited. 